\title{
New Pulsars from Arecibo Drift Scan Search
}

\author{
A. Somer \\ Astronomy Department 6 Radio Astronomy Laboratory, University of \\ California, Berkeley, $C A$
}

\begin{abstract}
.
We report the discovery of 3 pulsars, PSR J0030+0451, PSR J0711+09, and PSR J1313+09 that were found in a three dimensional (DM, period, position) search at $430 \mathrm{MHz}$ using the $305 \mathrm{~m}$ Arecibo telescope. PSR $\mathrm{J} 0030+0451$ is a nearby 4.8 -millisecond solitary pulsar. Spin and astrometric parameters are presented for the three new pulsars. We have measured significant polarization in the millisecond pulsar, PSR J0030+0451, over more than $50 \%$ of the period and use these data and also a morphological decomposition of the profile to briefly discuss magnetospheric models.
\end{abstract}

\section{Introduction}

From 1994 to 1995, during the time of the Gregorian Dome upgrade at the Arecibo Observatory, while the telescope had limited steerability due to construction, a joint effort by several collaborations was aimed at surveying the sky visible from Arecibo (declination range roughly $-1^{\circ}$ to $39^{\circ}$ ) in search of new pulsars. A total of 44 pulsars including 5 MSPs have been discovered so far by the other institutions (Foster et al. 1995, Camilo et al. 1996, Ray et al. 1996). Our search has an estimated sensitivity of $0.9 \mathrm{mJy}$ at $7 \sigma$ for long period, low DM, low zenith angle, high galactic latitude pulsars which is comparable to previous searches. In December 1997 we confirmed the presence of 3 new pulsars: J0030+0451 (4.8ms), J0711+09 (2.4s), and J1313+09 (0.85s) at the Arecibo Observatory using the Arecibo Berkeley Pulsar Processor and the Penn State Pulsar Machine (Backer et al. 1997, Foster et al. 1995). Follow up observations of the 3 new pulsars were conducted over a period of nearly 2 years from December 1997 through September 1999 at the Arecibo Observatory. 


\section{Analysis}

The data were cross correlated with a template, and the resulting TOAs analyzed using the TEMPO program (Taylor \& Weisberg 1989). Table 1 provides spin, astrometric and other parameters for each of the 3 new pulsars.

Table 1. Observed pulsar parameters.

\begin{tabular}{|c|c|c|c|}
\hline & $\mathrm{J} 0030+0451$ & J0711+09 & $\mathrm{J} 1313+09$ \\
\hline Right Ascension (2000) & $003027.4339(6)$ & $071136.74(2.00)$ & $131326.5(2.0)$ \\
\hline Declination (2000) & $045139.65(2)$ & $093140(30)$ & $093331(30)$ \\
\hline Galactic longitude & $113.1^{\circ}$ & $206.7^{\circ}$ & $320.4^{\circ}$ \\
\hline Galactic latitude & $-57.6^{\circ}$ & $8.8^{\circ}$ & $71.7^{\circ}$ \\
\hline Period(s) & $0.00486545320737(1)$ & $1.21409045(4)$ & $0.84893276(7)$ \\
\hline$\dot{P}\left(10^{15}\right) \mathrm{s} \mathrm{s}^{-1}$ & $1.0(2) \times 10^{-5}$ & $0.246(300)$ & $1.947(2000)$ \\
\hline Epoch (MJD) & 50984.4 & 50699.5 & 50984.5 \\
\hline $\begin{array}{l}\text { Dispersion Measure } \\
\left(\mathrm{pc} \mathrm{cm}^{-3}\right)\end{array}$ & $4.3326(1)$ & $46.15(200)$ & $12.0(1)$ \\
\hline Timing Data Span (MJD) & $50789-51277$ & $50789-51104$ & $50788-51080$ \\
\hline RMS timing residual $(\mu \mathrm{s})^{a}$ & 13 & 4500 & 1400 \\
\hline Flux $S_{400}(\mathrm{mJy})$ & $7.9(1)$ & $2.4(1)$ & $3.5(1)$ \\
\hline Flux $S_{1400}(\mathrm{mJy})$ & $0.6(1)$ & $>0.04(1)$ & $>0.16(1)$ \\
\hline DM distance $(\mathrm{pc})^{b}$ & 230 & 2450 & 780 \\
\hline$L_{400} \mathrm{mJy} \mathrm{kpc}^{2}$ & 0.4 & 14 & 2.1 \\
\hline Spectral index & $2.2 \pm .2$ & $<3.5$ & $<2.6$ \\
\hline Characteristic age (y) & $8 \times 10^{9}$ & $7 \times 10^{7}$ & $7 \times 10^{6}$ \\
\hline Magnetic field $(\mathrm{G})^{c}$ & $2.2 \times 10^{8}$ & $5.5 \times 10^{11}$ & $1.3 \times 10^{12}$ \\
\hline $\begin{array}{l}\text { Proper motion upper } \\
\text { limit }(\operatorname{arcsec} / \mathrm{yr})\end{array}$ & .060 & (none) & (none) \\
\hline
\end{tabular}

${ }^{a}$ RMS from 3 minute averages

${ }^{b}$ Model from Taylor \& Cordes, 1993

${ }^{c} B_{o}=3.2 \times 10^{19} \sqrt{P \dot{P}}$

\section{Millisecond Pulsar : PSR J0030+0451}

A pulsar displaying an interpulse like PSR J0030+0451 has two distinct possible geometries: nearly aligned, with the interpulse resulting from the second crossing of a wide-angle hollow "cone" of radiation, or orthogonal, with the two emission regions coming from opposite poles. We performed a dual frequency morphological study of the intensity profile to look for clues to the geometry of the system. Figure 1 depicts the Gaussian decomposition of the profile at each frequency, with the corresponding Gaussian parameters shown in Table 2 (Kramer et al. 1994, Kramer 1994). Conal emission is suggested by the narrowness of the $1400 \mathrm{MHz}$ model compared to the $430 \mathrm{MHz}$ data (panel b overlays the two). In addition, the amplitudes of the Gaussian components suggest that possibly all but component 2 are steep spectral index peaks, which may indicate that component 2 is a core component, and the rest are cone components.

The polarization data do not settle the question of whether the geometry is orthogonal or nearly aligned. Figure 2 shows an example of each. 


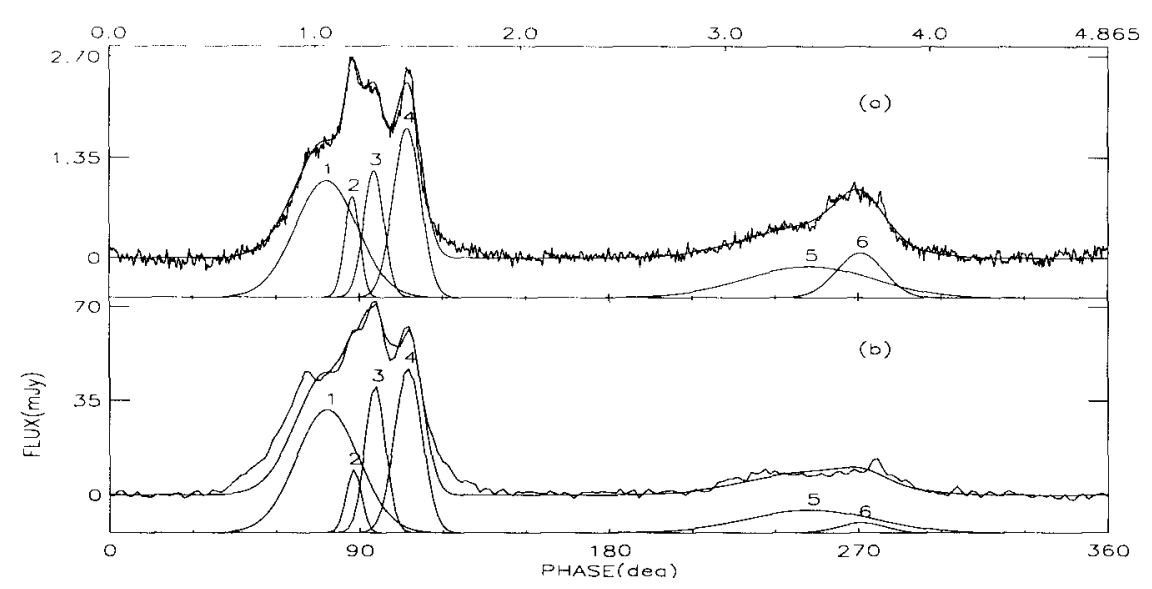

Figure 1. Decomposition of PSR J0030+0454 intensity profile into 6 components. (a) $1400 \mathrm{MHz}$ (b) $430 \mathrm{MHz}$. The best-fit Gaussian components at $1400 \mathrm{MHz}$ were only allowed to vary in amplitude in order to fit the $430 \mathrm{MHz}$ profile. (cf. Lorimer, "A Week in Review", these proceedings)

Of the 36 known MSPs in the disk of the galaxy, PSR J0030+0451 is the 9 th solitary MSP, i.e., it is not in a binary system. These objects present a unique problem in the standard evolutionary scenario of MSPs. If the neutron star is spun up via accretion by a mass transfer from a companion star, the companion must somehow be obliterated.

Table 2. Fitted Gaussian parameters for the 6 components in Figure 2. The amplitude of component 2 was arbitrarily set to 1.0 for both frequencies. All other amplitudes are relative to component 2.

\begin{tabular}{|l|r|r|r|r|r|r|}
\hline Peak \# & 1 & 2 & 3 & 4 & 5 & 6 \\
\hline Center (deg) & 78.74 & 88.13 & 95.88 & 107.85 & 253.106 & 271.45 \\
Width (deg) & 15.96 & 3.854 & 5.183 & 7.012 & 32.77 & 12.23 \\
Amplitude (430 MHz) & 1.95 & 1.00 & 2.34 & 2.61 & 0.771 & 1.26 \\
Amplitude (1400 MHz) & 1.15 & 1.00 & 1.25 & 1.67 & 0.473 & 0.442 \\
\hline
\end{tabular}

\section{Acknowledgements}

I'd like to thank the LOC for their financial support and for a wonderful conference! Many thanks to Michael Kramer for timing observations from the MPIfR 100-m telescope and for the use of his Gaussian fitting program, Alex Wolszczan for use of the Penn State Pulsar Processor, and Kiriaki Xilouris and Duncan Lorimer for extensive assistance in observations. 


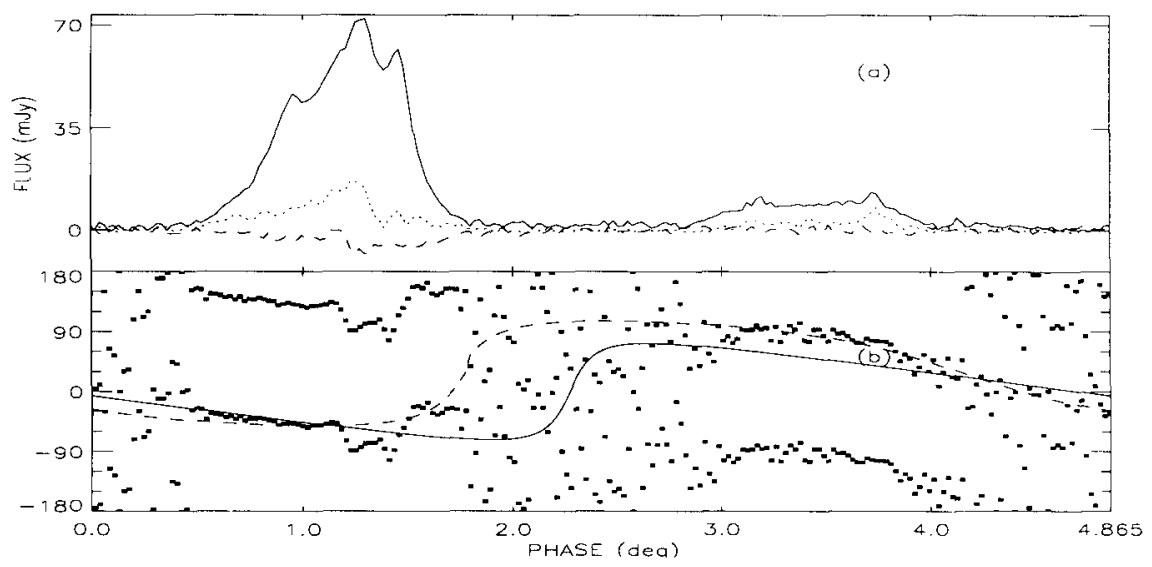

Figure 2. PSR J0030+0454 at $433 \mathrm{MHz}$. (a) The solid line shows intensity vs. phase. The dotted line shows linear polarization and the dashed line shows circular polarization. (b) Two RVMs are superimposed on the PPA data. The solid line demonstrates a possible nearly aligned rotator, plotted with $\alpha=8^{\circ}$ and $\beta=1^{\circ}$. The dashed line demonstrates a possible orthogonal rotator with values $\alpha=62^{\circ}$ and $\beta=10^{\circ}$.

\section{References}

Backer, D. C., Dexter, M. R., Zepka, A., Ng, D., Werthimer, D. J., Ray, P. S., \& Foster, R. S. 1997, PASP, 109, 61

Camilo, F., Nice, D. J., Shrauner, J. A., \& Taylor, J. H. 1996, ApJ, 469, 819

Foster, R. S., Cadwell, B. J., Wolszczan, A., \& Anderson, S. B. 1995, ApJ, 454, 826

Kramer, M. 1994, A\&AS, 107, 527

Kramer, M., Wielebinski, R., Jessner, A., Gil, J. A., \& Seiradakis, J. H. 1994, A\&AS, 107, 515

Ray, P., van Kerkwijk, M. H., Kulkarni, S. R., Prince, T. A., Sandhu, J. S., \& Nice, D. J. 1996, ApJ, 470, 1103

Taylor, J. H., \& Weisberg, J. M. 1989, ApJ, 345, 434

Taylor, J. H., \& Cordes, J. M. 1993, ApJ, 411, 674 